\title{
Dermatobia hominis misdiagnosed as abscesses in a traveler returning from Brazil to Denmark
}

\author{
Jonas Olsen ${ }^{\circledR}$, Peter Nejsum ${ }^{2,3}$, Gregor Borut Ernst Jemec ${ }^{1}$
}

\begin{abstract}
We present the case of a 62-year-old woman that consulted us for two boil-like lesions on her thighs after returning from a trip to São Paulo, Brazil, where she had swum in a freshwater lake. After consulting three specialist doctors and undergoing two antibiotic treatments, she was diagnosed with furuncular myiasis caused by Dermatobia hominis. The parasites were excised with no complications.
\end{abstract}

Keywords: dermatology, parasitology, diagnostics, dermoscopy, skin surgery

Received: 27 January 2017 | Returned for modification: 22 February 2017 |Accepted: 4 March 2017

\section{Case report}

A 62-year-old woman presented with two papules with small central ulcerations on her thighs after travelling to Brazil 2 months earlier. During a trip to the rural parts of São Paulo, Brazil, the patient experienced mosquito bite-like lesions, one on each thigh, after swimming in a freshwater lake. During the following 6 weeks, the lesions developed a boil-like appearance with central ulceration and pus oozing from the lesions, growing to $1.5 \mathrm{~cm}$ in diameter (Fig. 1a). During their development, the patient experienced paroxysmal shooting pains and itching. After returning home, she was examined first by her GP, who diagnosed the lesions as abscesses, obtained swabs of the lesions for bacterial cultures, and prescribed roxithromycine $150 \mathrm{mg}$ two times daily. Seven days later she was referred to the Department of Internal Medicine due to a lack of clinical response, where she was prescribed dicloxacilline $1 \mathrm{~g}$ three times daily for 7 days instead of roxithromycine based on identification of Staphylococcus aureus in bacterial cultures. All clinical examinations were normal except for the lesions, as were blood panels including eosinophils. At a follow-up examination 1 week later (8 weeks after onset) there was no clinical improvement, and, based on her travel history, the suspicion of tropical parasitic infection was raised. Her leishmaniasis antibody level was measured, and metronidazole 5 mg three times daily for 7 days was added to the treatment. The patient was referred to the Department of Dermatology for a punch biopsy. Dermoscopy revealed movement of a parasite in the ulcerations of the lesions (Fig. 1b). Furuncular myiasis was suspected, and surgical excision was performed with local anesthesia with lidocaine $20 \mathrm{mg} / \mathrm{ml}$. After anaesthetizing the skin, the respiratory end of the larvae protruded approximately $5 \mathrm{~mm}$ out of the lesions (Fig 2a). The excised material confirmed cutaneous myiasis with larvae measuring approximately $2 \mathrm{~cm}$ in length and $1 \mathrm{~cm}$ in width (Fig 2b). The patient suffered no sequelae. Histopathology revealed a cystic process containing the larva in the subcutis surrounded by a fibrotic capsule and chronic inflammation in the dermis.

DNA was extracted from the larvae using the MasterPure DNA Purifications Kit (Epicentre Biotechnologies) according to the manufacturer's protocol. The forward primer DhCOIF (5'-ATTTTCGGTGCTTGATCTGG-3') and reverse DhCOIR (5'-GAATTGGATCTCCTC-
CTCCTG-3') were designed using Primer 3 and used to amplify a $628 \mathrm{bp}$ fragment of the cox1 gene. Standard PCR conditions were applied with an annealing temperature of $60^{\circ} \mathrm{C}$. The PCR product was purified and sequenced in both directions by Macrogen Inc. in Seoul, South Korea. The sequence was found to be $99 \%$ identical to Dermatobia hominis from Brazil (Genbank accession nos. JQ246701 and AY463155) using a BLAST search (Genbank).

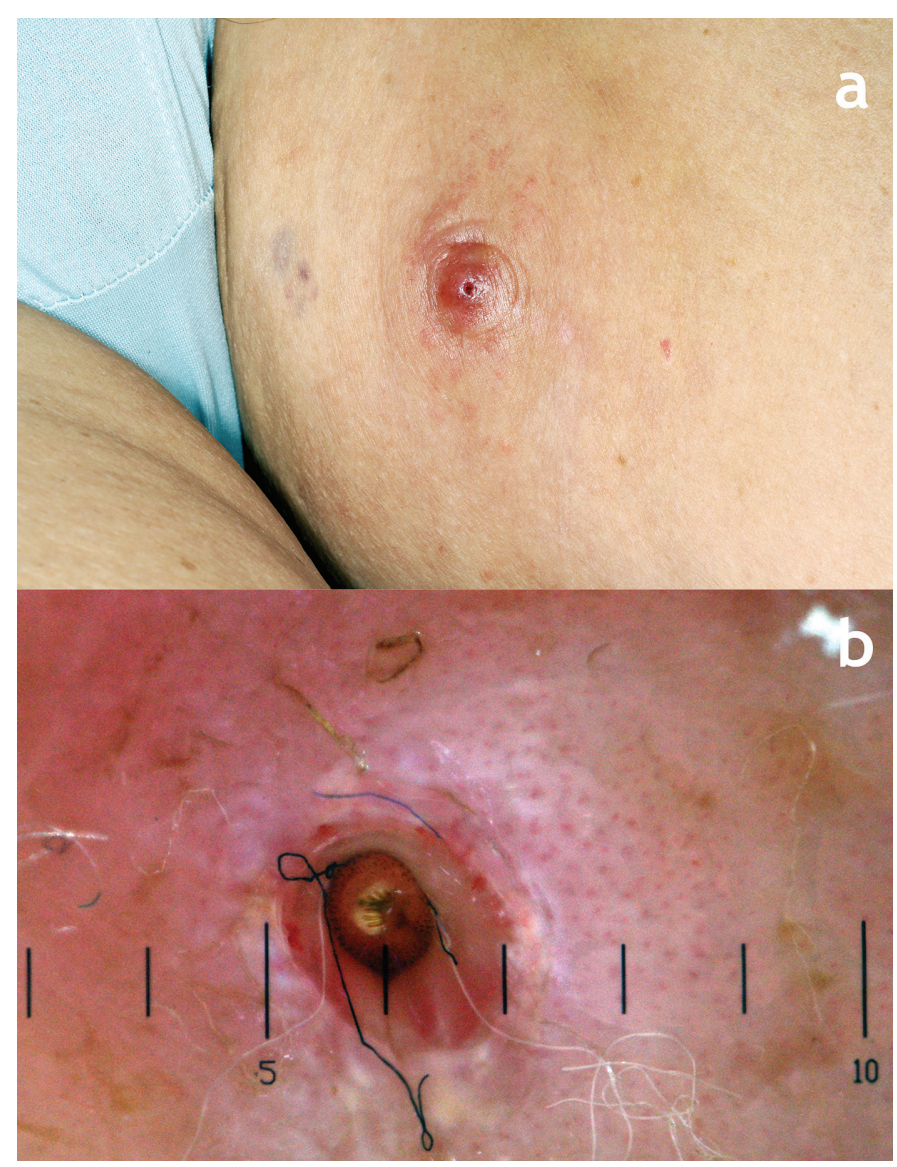

Figure 1 | Clinical presentation: a) Lesion on the left thigh between the quadriceps and adductor muscles at the time of diagnosis at the Department of Dermatology. Diameter of $1.5 \mathrm{~cm}$ and a small central ulceration. b) Dermoscopy of the lesion on the left thigh showing the central ulceration (diameter $3 \mathrm{~mm}$ ) with presence of the parasite's breathing tube. 


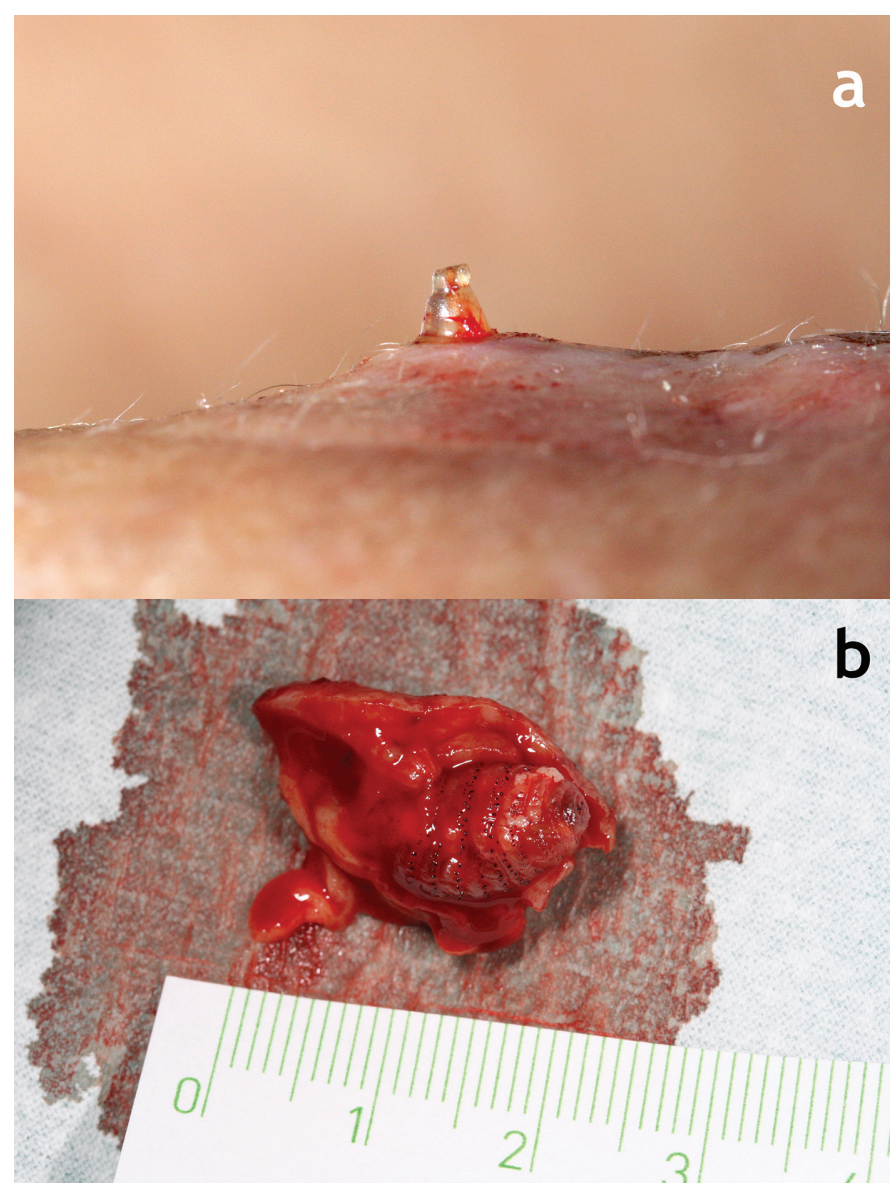

Figure 2 | During and after excision: a) Lesion on the right thigh after local anesthesia with injection of lidocaine $20 \mathrm{mg} / \mathrm{ml}$, showing the rear portion of the parasite protruding approximately $5 \mathrm{~mm}$ out of the lesion. b) Surgically removed larva, approximately $2 \mathrm{~cm}$ long and up to $1 \mathrm{~cm}$ wide. Characteristic rows of black spines can be seen along the widest part of the larva with the mouth parts on the right side of the larva. The skin surface is shown at the top of the excised material, in which the larva is positioned in a J-shape.

\section{Discussion}

D. hominis, the human botfly, is the most common cause of furuncular myiasis in Mexico, Central America, and South America
(1). It is transferred to cattle and humans by mosquitos and other bloodsucking insects that have eggs deposited underneath their bodies by the adult botfly. The eggs hatch when exposed to body heat and the larvae penetrate the skin (2). The larvae feed inside a subdermal cavity for 5 to 10 weeks, and undergo a development of three instars. The third instar begins after approximately 4 weeks, in which the larvae develop a cylindrical shape and a length of up to $23 \mathrm{~mm}$ (3). The patient was infected approximately 8 weeks before being diagnosed and, combined with the size and morphology of the larvae (Fig. 2b), this suggests that they were mature third-instar larvae. According to the Centers of Disease Control and Prevention, covering bare skin, using insect repellant, using mosquito nets, and ironing clothes may prevent myiasis (4).

The challenge of diagnosing the parasite earlier could have been accomplished by either dermoscopy or ultrasound. Dermoscopy may reveal the breathing parts of the parasite, confirming the presence of larvae (5). Ultrasound has been shown to reveal an oval structure with a hypoechoic rim and hyperechoic center, spontaneous movement, and peripheral blood flow (6).

The treatment options for $D$. hominis larvae are either manual extraction or surgical excision. Before performing manual extraction, asphyxiation of the parasite is necessary by the application of occluding substances (e.g., nail polish, petroleum jelly, etc.) and then carefully pulling out the larvae. The choice of treatment depends on the size of the larvae and anatomical location; particularly large third-instar larvae are hard to remove without surgical excision (7). This is due to the risk of damaging the larvae and leaving debris, causing foreign-body reactions or secondary infections (1).

The diagnosis was not made until the patient had undergone multiple treatments with inappropriate antibiotics, implying the need for further education in diagnosing tropical parasites in non-endemic areas, particularly due to the increasing popularity of travel in the tropics.

\section{References}

1. Zammarchi L, Viligiardi R, Strohmeyer M, Bartoloni A. Dermatobia hominis: small migrants hidden in your skin. Ann Dermatol. 2014;26:632-5.

2. Robbins K, Khachemoune A. Cutaneous myiasis: a review of the common types of myiasis. Int J Dermatol. 2010;49:1092-8.

3. Villalobos G, Vega-Memije ME, Maravilla P, Martinez-Hernandez F. Myiasis caused by Dermatobia hominis: countries with increased risk for travelers going to neotropic areas. Int J Dermatol. 2016;55:1060-8.

4. Centers of Disease Control and Prevention. cdc.gov: Parasites - myiasis: Centers of Disease Control and Prevention; 2014 [cited 24 February 2017]. Available from: https://www.cdc.gov/parasites/myiasis/faqs.html.
5. Bernardes Filho F, Martins G, Barbara EF, Paiva ML, Coelho Filho RL, Nery JA. Dermoscopy as an auxiliary tool for the diagnosis of furuncular myiasis. An Bras Dermatol. 2014;89:663-5.

6. Bouer M, Rodriguez-Bandera Al, Albizuri-Prado F, Lobos A, Gubeling W, Wortsman X. Real-time high-frequency colour Doppler ultrasound detection of cutaneous Dermatobia hominis myiasis. J Eur Acad Dermatol Venereol. 2016;30:e180-e1.

7. Smith SM. Treating infestations of the human botfly, Dermatobia hominis. Lancet Infect Dis. 2015;15:512. 